\title{
Differences of inter-canine distance on dental cast model, wax impression tracing, radiograph of dental impression tracing, and dental cast tracing methods of human bite mark
}

\author{
Fidya $^{1 *}$, Astika Swastirani ${ }^{2}$ \\ ${ }^{1}$ Department of Oral Biology, Faculty of Dentistry Brawijaya University, Indonesia \\ ${ }^{2}$ Department of Oral Medicine, Faculty of Dentistry Brawijaya University, Indonesia
}

\begin{abstract}
Introduction: Bite marks are unique to each, differing between one person and another. Several methods to analyse bite mark have been developed during certain periods. However, a standard method to analyse and evaluate the bite mark anatomic variations has not been developed. The objective of this research was to identify the differences of the inter-canine distance on dental cast model, wax impression tracing, radiograph of dental impression tracing, and dental cast tracing methods of the human bite mark. Methods: The subject of the research was as much as 30 consecutive dental cast model, wax impression tracing, radiograph of dental impression tracing, and dental cast tracing for both maxilla and mandible. Measurements performed on each group were conducted in the inter-canine areas using a Vernier calliper. The gold standard that used as a comparator was a dental cast model group on both maxilla and mandible. Comparison of the measurement results was carried out and analysed using a oneway ANOVA test and Tukey LSD test. Results: The average sizes of maxilla and mandible were $37.93 \mathrm{~mm}$ and $31.70 \mathrm{~mm}$ for dental cast model; $37.93 \mathrm{~mm}$ and 32.83 for wax impression tracing; $38.20 \mathrm{~mm}$ and $31.70 \mathrm{~mm}$ for radiograph dental impression tracing; and $36.65 \mathrm{~mm}$ and $30.76 \mathrm{~mm}$ of dental cast tracing. The significance value of the maxilla was $p=0.008(p<0.05)$, and the significance value of mandible was $p=0.000(p<0.05)$. Conclusion: The inter-canine distance of radiograph tracing group is found to be most similar with dental cast group as gold standard both in the maxilla and the mandible.
\end{abstract}

Keywords: Bite mark, dental cast, wax impression, radiograph, tracing

p-ISSN 1979-0201, e-ISSN 2549-6212; Available from: http://jurnal.unpad.ac.id/pjd/article/view/16614

DOI: 10.24198/pjd.vol31no1.16614

Submission: Apr 30, 2018; Reviewed: Oct 15, 2018; Resubmit for review: Jan 7, 2019; Accepted: Mar 11, 2019; Published online: Mar 29, 2019

\section{INTRODUCTION}

The tooth is the hardest human tissue with chemical element stability. It lasts long and endures towards environmental pressure, aside from its static nature. It does not experience diagenesis. In other words, it does not decompose although being buried for a long time. These natures make the tooth can be studied, examined, and measured evolutionally. ${ }^{1}$ 
Odontometrics, the measurement of any teeth group, is a useful information for identification process. It can be used as references in several dental treatment procedure and forensic odontology examination. Size of teeth has an important role in indicating variations of activities that deals with occlusion, determining the frequency of dental and bone tissue anomaly in orthodontic treatment application, and determining sex. ${ }^{2}$

All teeth, both primary and permanent teeth, have the same structure in terms of email, dentine, and pulp. But teeth vary in shape, size, and distance for each individual. This makes teeth have distinctive arrangements for each. ${ }^{3}$ Teeth have different morphological features, so no two morphological teeth are the same; they are unique in each person. This uniqueness is determined by genetic factors. Dental trait is a dental characteristic that shows variations of anatomical morphology that are always associated with dental anthropology and forensic odontology.

Dental variations help compare antemortem and post-mortem data for identification because the characteristics of teeth are unique and different from one individual to another. This characteristic help to classify and identify population, as well as individuals. Dental trait has a positive potential in identification, as DNA and fingerprints. It helps estimate biological profiles, such as age, gender, and race of individuals. This analysis forms the basis for identification process in humans. ${ }^{4}$

One method of identification is using teeth, which is through bite mark. The mark is highly individualistic and distinctive in nature. ${ }^{3}$ Bite marks are patterns produced by human and animal bites. It has unique characteristics in each person. There are no similarities of patterns from one individual to another, even between identical twins. ${ }^{5}$ The characteristics of tooth structure such as distance, angle, missing teeth, and dental restorations can produce different bite marks. ${ }^{6}$

Bite marks are physical changes in body parts caused by contact, or interdigitation between the upper teeth with the lower teeth, with human or animal teeth, so that a tissue structure is injured. Bite marks can be found in any part of body in humans. ${ }^{7}$ Two important points that should be noted from bite marks are dental arches and traits. Although several methods for analyzing dental trait from bite mark have been developed, the standard method for analyzing and evaluating anatomic variations has not yet been developed. ${ }^{8}$ The process of comparing bite marks with suspect's dental arrangements is done through analysis and measurement of teeth size, shape, and position. Bite mark analysis can be done through manual and computerized study model, bite registration wax, xenograft, and radiology. ${ }^{9}$ This research was aimed to identify the differences of the inter-canine distance on dental cast model, wax impression tracing, radiograph of dental impression tracing, and dental cast tracing methods of the human bite mark.

\section{METHODS}

The design of this study was observational using dental metric measurement. The samples of this study were dental cast model (Model group), wax impression tracing (Wax Tracing), radiograph of dental impression tracing (Radiograph Tracing), and dental cast tracing (Model Tracing) from human bite mark. The measurement of the bite mark will resulted in deformation depending on variations in tissue structure, dehydration, and photographic techniques but the relationship between adjacent teeth remained the same. If the position of the teeth remained constant, identification can be done. The constant position of the teeth can be measured through the width of the inter-canine distance. ${ }^{10}$

For model group, the measurement was directly measured using Vernier caliper from distal right canine to distal left canine. For the other groups the measurement was conducted in tracing result from wax group, radiograph group, and tracing model group both in maxillary and mandible. The cast model was from 30 impression of 30 subject research. The number of subjects obtained by Central Limit theory. The subjects were given information before and asked to fill inform consents for bite-mark printing. Measurements in the study model were performed using Vernier caliper from left to right caninus on the distal side.

In this research, inter-observer measurements were performed by two observers. The result of this measurement was then 
compared with the independent t-test to analyse the significance of the difference. The result of this measurement was not significantly different $(p>0.05)$ between two observers, so one of the data can be used. Measurement in the dental cast model were carried out directly using the Vernier caliper at the distal right canine to the distal left canine. The measurement results were then recorded on the maxilla and mandible.

Measurement of wax impression tracing was performed by asked the research subjects to bite red wax plate that usually use for making denture. Then, infection control was performed on the results of the bite-mark printing. The tracing was done on impression wax using transparent paper and pencil. The result of tracing was measured from distal right canine to distal left canine.

The bite on registration wax was given dental temporary filling material (dental cavit) according to the resulting impression. The wax was $x$-rayed on $5 \times 7 \mathrm{~cm}$ of occlusal film. The radiographic images were traced using transparent paper with pencil. Measurement of tracing result from distal canine right to distal canine left. Tracing on the model on transparent paper and measuring the results of tracing using pencil on the maxilla and mandible from the distal canine right to the distal left canine.

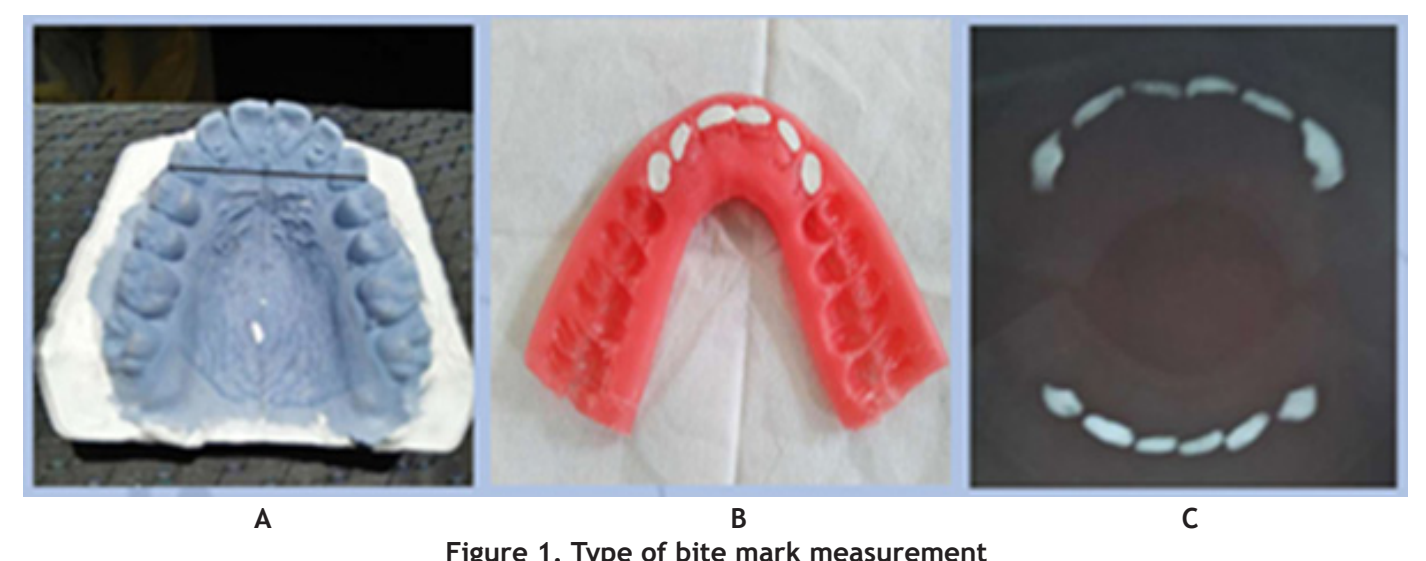

( $\mathrm{A}=$ dental cast model; $\mathrm{B}=$ wax impression; $\mathrm{C}=$ radiograph dental impression)

The inter-canine distance in maxilla and mandible were measured by using Vernier caliper from distal right canine to distal left canine. The result of inter-canine distance was calculated on the Model group, Wax Tracing group, Radiograph Tracing group, and Model Tracing group. The data were tabulated, and they were tested using oneway ANOVA and Tukey LSD test.

The data from the measurement results were tabulated and tested by Shapiro-Wilk test and Levene test. Both test results in $p>0.05$, which means that the maxillary and mandibular measurements data are normally distributed and homogeneous. The next test was one-way ANOVA, which was performed to see the differences between groups. The results of the ANOVA test on maxilla are significant at $0.008(p<0.05)$, which means significant differences between groups are found. The next was the Tukey LSD test, which was carried out to see the differences between groups.

\section{RESULTS}

The measurement results in this study include maxilla and mandible inter-canine distance of the model group, the wax tracing group, the radiograph tracing group, and the dental model tracing group. The mean of the measurement using the four techniques above is presented in Table 1.

The results of one-Way ANOVA test were showed significant differences among four group in maxilla $p=0.008(p<0.05)$ and mandible $p=$ $0.000(p<0.05)$. Tukey LSD test result for maxilla showed significant differences $(p<0,05)$ between Model group and Model Tracing group; Wax group and Model Tracing group; Radiograph Tracing group and Model Tracing group and not significant different $(p>0.05)$ between Model group and Wax Tracing group; Model group and Radiograph Tracing group; Wax Tracing group and Radiograph Tracing group. 
Tukey LSD test result for mandible showed significant differences $(p<0.05)$ between the Model group and the Wax Tracing group; the Wax Tracing group and the Model Tracing group; the Wax Tracing group and the Model Tracing group; and not significant difference $(p>0.05)$ between the Model group and the Radiograph Tracing group; the Model group and the Model Tracing group; the Radiograph Tracing group and the Model Tracing group.

Table 1. The mean of model group, wax tracing group, radiograph tracing group, and dental model tracing group measurement of maxilla and mandible $(\mathrm{mm})$

\begin{tabular}{llcccccccc}
\hline \multirow{2}{*}{ No Group } & \multicolumn{4}{c}{ Maxilla } & \multicolumn{4}{c}{ Mandible } \\
\cline { 2 - 10 } & & Sum & Mean & SD & p & Sum & Mean & SD & P \\
\hline 1 & Model & 30 & 37.93 & 1.79 & 0.008 & 30 & 31.03 & 1.67 & 0.000 \\
2 & Wax Tracing & 30 & 37.93 & 1.64 & & 30 & 32.83 & 1.78 & \\
3 & Radiograph Tracing & 30 & 38.20 & 1.95 & & 30 & 31.70 & 1.49 & \\
4 & Model Tracing & 30 & 36.65 & 2.07 & & 30 & 30.76 & 2.46 & \\
\hline
\end{tabular}

\section{DISCUSSION}

Bitemarks are caused by teeth or a combination of teeth and other parts of the mouth. The scientific foundation for bitemark analysis roots in the premise of human teeth individuality, the belief that no two human teeth are the same in terms of size, shape and structure. Bitemark analysis is based on assumptions about the uniqueness of human teeth, but it is almost impossible to match bitemarks with specific teeth. ${ }^{11}$ The most commonly used comparison method in bite mark analysis is creating overlays, which are transferring bitemarks to certain media. The results of oneway ANOVA test in this result showed significant differences $(p=0.008)$ among maxillary groups and $(p=0.000)$ mandibular groups, that indicating differences in the value generated by each group.

In the process of identification, determining of bitemark depends on the severity and anatomical location, because of that, the results obtained in this process are subjective in everyone. Like fingerprints and DNA, bitemarks are unique, in terms of distance and angle between teeth, missing teeth, filling, and types of dental care, in each person. ${ }^{11}$ The process of benchmarking bitemark and suspect's teeth includes analysis and measurement of teeth size, shape, and position. Human incision produces a rectangular shape, while the canine produces a triangular one..$^{12}$ In this study the size of inter-canine distance could be measured using all methods. There are variations between the four methods in determining intercanine distance in this research. This might occur as a subjective error that occurs when tracing uses the hand. Bitemark can be analyzed using several techniques that are direct or indirect. Direct technique is measurements on the bite in anatomy of the body or objects used, while the indirect technique uses media like transparent paper that is used to move bite mark for observation. Tracing on paper can be carried out on cast models, wax impression, and radiographs dental impression that have been filled with materials that reflect the radiopaque projection on the film. ${ }^{13}$

The results of Tukey LSD test on the maxilla group showed no significant difference in measurement results between the dental cast model group and wax tracing group; and between the dental cast model and radiograph tracing model. The results of mandible measurements showed no significant difference on the comparison between model group and radiograph tracing group; and between model group and model tracing group. result is because of the gap of the mesial distal edge of 6 anterior teeth of the maxilla is clearly visible, so the distance from the right to the left distal canine can be measured easily. The wax impression method produces bite prints with a rotation angle detected properly. This method allows the results to cover all areas of the tooth with well-documented angles and contact points.

The significant differences result was found in maxilla measurement results of the dental cast tracing group because the mesial and distal edges of 6 anterior teeth were difficult to see, thus determining the distal edges of the right and left canine was more difficult. The previous research gave the best assasing in different tooth. The hand 
tracing wax group in right second incisivus and left canine; and radiograph wax impression group in right canine. ${ }^{13}$ In maxillary measurements, intergroup test results show that the measurement using hand tracing method have significant results $(p<0.05)$. This suggests that this method is insufficient in representing the actual bite marks formation since it is a replica.

The hand tracing method allows the occurrence of subjective errors when tracing images from the model which make less accurate results. ${ }^{13}$ In addition, tracing methods rely heavily on the ability of the researchers in moving bite mark formation from the original into images.

The significant differences result was found in mandible measurement results $(p<0.05)$ between Model group and Wax group; Wax group and Tracing group; Wax group and Tracing group. The previous research gave the best assasing in different tooth. The hand tracing models group in right and left canines; hand tracing wax group in first and second right incisivus; and radiograph wax impression group in first and second left incisivus. ${ }^{13}$ This result is because of the superimposed maxilla and mandible bite marks that make the distal edges of the right and left canine teeth are difficult to determine. In mandible measurement, intergroup test results showed that the measurement using wax tracing method gave significant results. In the wax method, the tooth is pressed in the wax, so that the printed area becomes clear, including the lines of the angle and the contact point the tooth. ${ }^{13}$

This is possible because of the mobile position of mandible, which causes shifting during the printing process, so the bite on the wax is affected. In this research the inter-canine distance in radiograph tracing group was found most similar with dental cast group as gold standard both in maxilla and mandible.

\section{CONCLUSION}

There are differences among four groups. In the maxilla, the inter-canine distance of the tracing cast model group has significant difference with other groups; while in the mandible, the intercanine distance of the wax impression group has significant difference with other groups. The inter-canine distance of radiograph tracing group is found to be most similar with dental cast group as gold standard both in the maxilla and the mandible, thus the radiograph tracing method can be used for identification in bite mark analysis.

\section{REFERENCES}

1. Artaria MD. Antropologi dental. Edisi 1. Yogyakarta: Graha Ilmu. 2009.9-13p.

2. Duraiswamy P, Tibdewal H, Patel K, Kumar S, Dhanni C, Kulkarni S. Sex determination using mandibular canine index in optimal-fluoride and high-fluoride areas. J Forensic Dent Sci. 2009; 1(2):99-103.

3. Krishan K, Kanchan T, Garg AK. Dental evidence in forensic identification - an overview, methodology and present status. Open Dent J. 2015; 1(9):250-6.

4. Barsley R, Freeman A, Metcalf R, Senn D, Wright F. Bitemark analysis. J. Am. Dent. Assoc. 2012;143(5):444.

5. Mahajan A, Batra APS, Khurana BS, Seema, Kaur J. Role of bitemark analysis in indentification of a person. GJMPH. 2012; 1(1):56-9.

6. Kaur S, Krishan, K., Chatterjee, P.M., Kanchan, T. Analysis and identification of bite marks in forensic casework. Oral Health Dent Manag. 2013; 12(3):127-31.

7. Hinchliffe J. Forensic odontology, Part 1. Dental identification. BDJ Online. 2011; 210(5): 219-24.

8. Maloth S, Ganapathy KS. Comparison between five commonly used two-dimensional methods of human bite mark overlay production from the dental study casts. Indian J Dent Res. 2011; 22(3): 493. DOI: 10.4103/0970-9290.87079

9. Stols $\mathrm{G}$, Bernitz H. Reconstruction of deformed bite mark using affine transformations. J Forensic Sci. 2010; 55(3): 784-7. DOI: 10.1111/j.1556-4029.2010.01337.x

10. Verma AK, Kumar S, Bhattacharya $S$. Identification of a person with the help of bite mark analysis. J Oral Biol Craniofac Res. 2013. 3(2): 88-91. DOI: 10.1016/j.jobcr.2013.05.002

11. Patil S, Rao R, Raj AT. A Comparison between manual and computerized bite-mark analysis. J Adv Oral Res. 2013. 4(3): 1-5. DOI: 10.1177/2229411220130301

12. Bhargava K, Bhargava D, Rastogi P, Paul $M$, Paul R, Jagadeesh HG, et al. An overview of 
bite mark analysis. J Ind Acad Forensic Med. 2012. 34(1): 61-6.

13. Pallam NK, Boaz K, Natrajan S, Raj M, Manaktala N, Lewis AJ. Computer-based method of bite mark analysis: A benchmark in forensic dentistry? J Forensic Dent Sci. 2016. 8(1): 32-9. DOI: 10.4103/0975-1475.176944 